\title{
Strong Exciton-Photon Coupling with Colloidal Nanoplatelets in an Open Microcavity
}

\author{
Lucas C. Flatten, ${ }^{*}{ }^{\dagger}$ Sotirios Christodoulou, ${ }^{\dagger} \perp$ Robin K. Patel, ${ }^{\dagger}$ Alexander Buccheri, ${ }^{\dagger}$ David M. Coles, ${ }^{\dagger}$ \\ Benjamin P. L. Reid, ${ }^{\S}$ Robert A. Taylor, ${ }^{\S}$ Iwan Moreels, ${ }^{\dagger}$ and Jason M. Smith ${ }^{* \dagger}$ \\ ${ }^{\dagger}$ Department of Materials, University of Oxford, Oxford OX1 3PH, United Kingdom \\ ${ }^{\ddagger}$ Nanochemistry Department, Istituto Italiano di Tecnologia, Via Morego 30, IT-16163 Genova, Italy \\ ${ }^{\S}$ Department of Physics, Clarendon Laboratory, University of Oxford, Oxford OX1 3PU, United Kingdom \\ ${ }^{\perp}$ Department of Physics, University of Genoa, Via Dodecaneso 33, IT-16146 Genova, Italy
}

\section{Supporting Information}

\begin{abstract}
Colloidal semiconductor nanoplatelets exhibit quantum size effects due to their thickness of only a few monolayers, together with strong optical band-edge transitions facilitated by large lateral extensions. In this article, we demonstrate room temperature strong coupling of the light and heavy hole exciton transitions of CdSe nanoplatelets with the photonic modes of an open planar microcavity. Vacuum Rabi splittings of $66 \pm 1 \mathrm{meV}$ and $58 \pm 1 \mathrm{meV}$ are observed for the heavy and light hole excitons, respectively, together with a polariton-mediated hybridization of both transitions. By measuring the concentration of platelets in the film, we compute the transition dipole moment of a nanoplatelet exciton to be $\mu=(575 \pm 110) \mathrm{D}$. The large oscillator strength and fluorescence quantum yield of semiconductor nanoplatelets provide a perspective toward novel photonic devices by combining polaritonic and spinoptronic effects.
\end{abstract}

KEYWORDS: Nanoplatelets, strong coupling, room-temperature, polaritons, microcavity
$S^{\text {thom }}$ trong light-matter coupling results in the formation of new eigenstates, called polaritons, that consist of an admixture of the uncoupled states. Such coupling, well-known from atomic physics, was first demonstrated for an excitonic solid state system at cryogenic temperatures in GaAlAs/AlAs quantum wells. ${ }^{1}$ Room temperature exciton-polariton formation has since been demonstrated in organic molecules, ${ }^{2,3}$ wide band gap semiconductors such as $\mathrm{GaN}^{4,5}$ and $\mathrm{ZnO}$, 6,7 and more recently in two-dimensional materials. ${ }^{8,9}$ Strong coupling of colloidal nanocrystals in the form of quantum dots has been shown in evanescently coupled bilayer microcavities. ${ }^{10}$ The incorporation of polaritons in optoelectronic devices allows intriguing technological advances such as threshold-less polariton lasing, ${ }^{11,12}$ engineered polariton bistability, ${ }^{13}$ and a feasible route to interesting phenomena such as polariton condensation, superfluidity, and vortex formation. ${ }^{14,15}$

Most of these systems require cryogenic temperatures and complex device fabrication. Recent advances in nanoparticle synthesis have reinforced the prospects for improving on these systems, allowing more facile polariton implementations at room temperature, with processing steps such as printing of the active layer. Prominent examples for such materials are colloidal nanoparticles, which are the subject of active research due to their relatively simple chemical preparation and precisely tunable spectral properties. ${ }^{16,17}$ Zero-dimensional quantum $\operatorname{dots}^{18}$ and one-dimensional nanorods ${ }^{19}$ are ubiquitous in the field of nanophotonics. Interestingly, progress in chemical synthesis has allowed the preparation of colloidal, atomically thin, quasi-two-dimensional nanoplatelets (NPLs) ${ }^{20}$ with remarkable spectral properties such as narrow absorption and emission line widths at room temperature $(<40 \mathrm{meV})$ and quantum yields close to $50 \% .^{21,22}$ The large coherence area of the exciton causes an increased oscillator strength and fast exciton recombination rates down to picosecond values, which exceed those observed in traditional quantum dots by three orders of magnitude. ${ }^{23}$ Indeed, it is this large oscillator strength and the large exciton binding energy of $R_{\mathrm{exc}} \approx 100-300 \mathrm{meV}^{23}$ that make semiconducting nanoplatelets good candidates for entering the strong exciton-photon coupling regime at room temperature.

Here we give the first experimental evidence for polariton formation at room temperature with quasi-two-dimensional NPLs. We use a planar microcavity environment to create photonic modes of sufficient intensity to cause reversible coupling to the exciton dipole moments. Our experiment allows for in situ tunability of the photon-exciton coupling by variation of the cavity length. ${ }^{9}$ We analyze the polariton dispersion and probe the state population after off-resonant, optically pumped excitation. These results establish polariton

Received: August 15, 2016

Revised: October 7, 2016

Published: October 14, 2016 


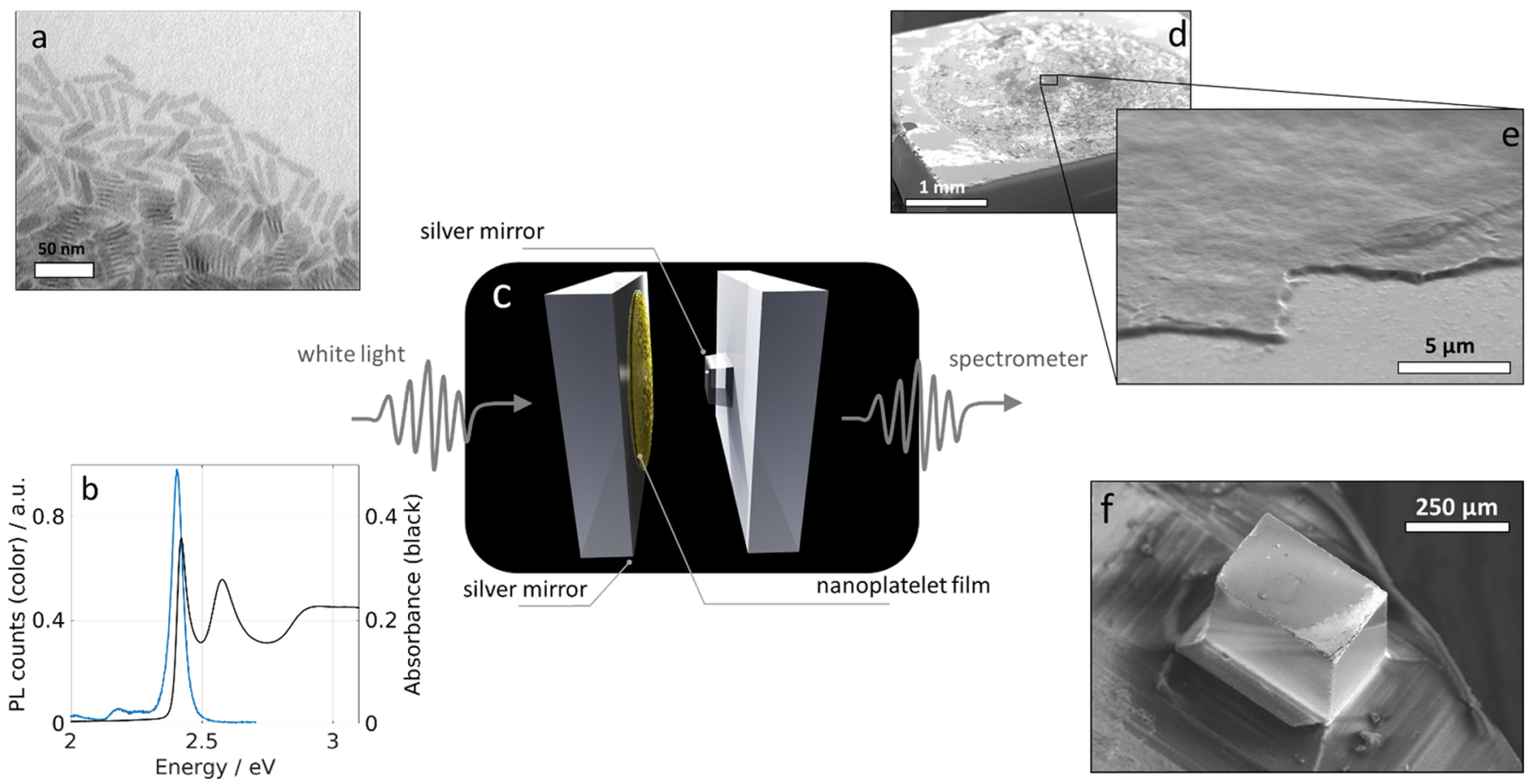

Figure 1. CdSe nanoplatelets in an open microcavity: (a) TEM image of dispersed CdSe nanoplatlets. Some stacked platelets are visible toward the bottom laying sideways. (b) Photoluminescence (color) and absorbance (black) of a CdSe nanoplatelet film deposited by dropcasting from solution. (c) Experimental setup: open-access microcavity consisting of two opposing, freely positionable silver mirrors. (d) SEM micrograph of silver mirror (left side of cavity) with (e) dropcast nanoplatelet film. (f) SEM micrograph of fused silica plinth coated with $50 \mathrm{~nm}$ of silver (right side of cavity).

formation in colloidal NPLs, a technological feature that could be used in broad fields such as laser and semiconductor physics.

The microcavity consists of two semitransparent silver mirrors ( $\sim 90 \%$ reflectivity) thermally evaporated onto fused silica substrates. One of the cavity mirrors is on a raised plinth of dimensions $200 \mu \mathrm{m}$ by $300 \mu \mathrm{m}$. A concentrated nanoplatelet dispersion is dropcast onto one of the mirrors and forms a dense nanoplatelet film approximately $700 \mathrm{~nm}$ thick (see Figure 1). The mirrors are brought to within $2 \mu \mathrm{m}$ of each other to form the optical cavity. The cavity length, and hence the cavity photon energy, can be scanned in situ with a piezoelectric actuator attached to one of the cavity mirrors (for details on the experimental setup, see Supporting Information). It is thus possible to access the whole polariton dispersion with different degrees of hybridization between heavy and light hole excitons.

The optical absorption spectrum of the nanoplatelets displayed in Figure 1, panel b reveals two transitions, which correspond to the lower energy heavy hole (hh) exciton with $E_{\mathrm{hh}} \approx 2.42 \mathrm{eV}$ and the higher-energy light hole (lh) exciton with $E_{\mathrm{lh}} \approx 2.56 \mathrm{eV}$. These states have binding energies of $R_{\mathrm{hh}}=$ $(178 \pm 34) \mathrm{meV}$ and $R_{\mathrm{lh}}=(259 \pm 3) \mathrm{meV}$, respectively. ${ }^{23}$

To probe the mode structure of the strongly coupled nanoplatelet-cavity system, the cavity length $L$ is varied, which leads to a shift in the energy of the cavity mode by $\Delta E_{\mathrm{c}}=E_{\mathrm{c}} \frac{\Delta L}{L}$, where $E_{c}=\frac{q h c}{2 L}$ is the energy of the original cavity mode with longitudinal mode number $q$ and $\Delta L$ the variation in cavity length.

The cavity length dependent optical transmission of the nanoplatelet-cavity system is shown in Figure 2, panel a. For the maximum cavity length of $L \approx 1.62 \mu \mathrm{m}$, a transmission peak corresponding to the lower polariton branch (LPB) is visible at $2.3 \mathrm{eV}$, which moves to higher energy with reduction of the cavity length (note the reversed $x$-axis of $2 \mathrm{a}$ ). As the LPB peak approaches the energy of the $\mathrm{hh}$ exciton at $2.42 \mathrm{eV}$, a second transmission peak, corresponding to the middle polariton branch (MPB), of energy $2.45 \mathrm{eV}$ appears above $E_{\mathrm{hh}}$. The LPB and MPB energies display an anticrossing about $E_{\mathrm{hh}}$. As the cavity length is decreased further, a third higher energy transmission peak above $E_{\mathrm{lh}}$ appears (the upper polariton branch (UPB)), which undergoes an anticrossing with the MPB about $E_{\text {lh }}$.

This system of one cavity mode simultaneously coupled to two excitonic transitions is described by the Hamiltonian:

$$
\begin{aligned}
& H=\quad E_{\mathrm{c}} b^{\dagger} b+E_{\mathrm{hh}} x_{\mathrm{hh}}^{\dagger} x_{\mathrm{hh}}+E_{\mathrm{lh}} x_{\mathrm{lh}}^{\dagger} x_{\mathrm{lh}}+ \\
& V_{\mathrm{hh}}\left(b^{\dagger} x_{\mathrm{hh}}+\text { c. c. }\right)+V_{\mathrm{lh}}\left(b^{\dagger} x_{\mathrm{lh}}+\text { c. c. }\right)
\end{aligned}
$$

where $V_{\mathrm{hh}}$ and $V_{\mathrm{lh}}$ are the interaction potentials between the cavity mode and heavy and light hole excitons. In addition, $b$, $x_{\mathrm{hh}}$ and $x_{\mathrm{lh}}$ are the photon, hh exciton, and lh exciton annihilation operators, respectively. In the stationary case, the system can be reduced to

$$
H|\Psi\rangle=\left(\begin{array}{ccc}
E_{c} & V_{\mathrm{hh}} & V_{\mathrm{lh}} \\
V_{\mathrm{hh}} & E_{\mathrm{hh}} & 0 \\
V_{\mathrm{lh}} & 0 & E_{\mathrm{lh}}
\end{array}\right)\left(\begin{array}{l}
\alpha \\
\beta \\
\gamma
\end{array}\right)=E|\Psi\rangle
$$

Here the state $|\Psi\rangle$ is defined by the three coefficients $\alpha, \beta$, and $\gamma$, which quantify the contribution of photon, hh exciton, and lh exciton, respectively.

By using the known values of $E_{\mathrm{hh}}, E_{\mathrm{lh}}$, and $E_{c}$ and treating $V_{\mathrm{hh}}$ and $V_{\mathrm{lh}}$ as free variables, we fit eq 2 to the observed polariton dispersion. At the crossing between unperturbed cavity mode and exciton energy, the polaritons are half-light, half-matter quasi-particles. The splitting at this point is the Rabi splitting, which corresponds to twice the interaction potential $\hbar \Omega_{\mathrm{hh}(\mathrm{hh})}=$ 

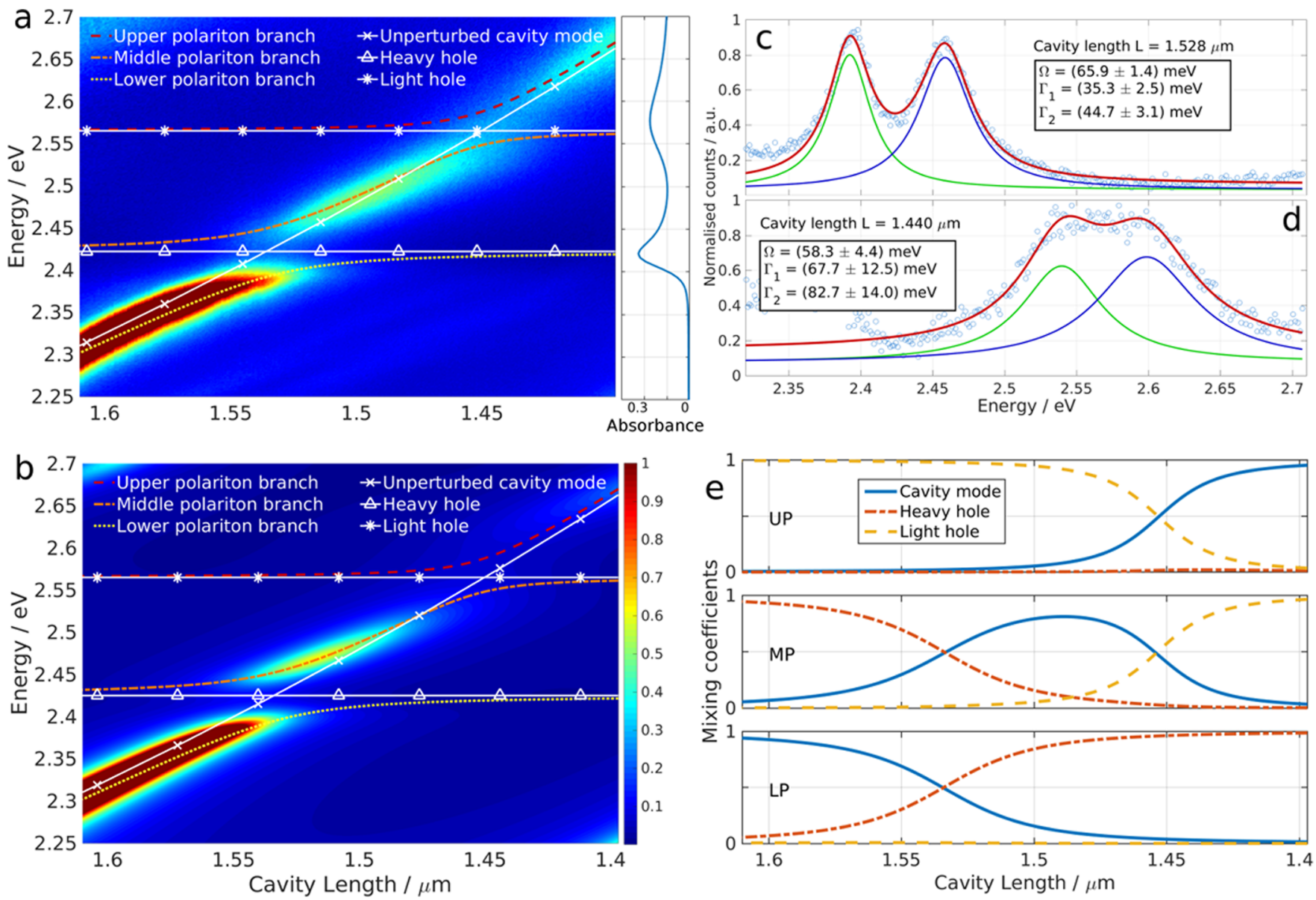

Figure 2. Normalized transmission spectra as cavity length is varied, obtained (a) experimentally and (b) by transfer-matrix calculations. The bare light and heavy hole exciton energies and the unperturbed cavity mode are overlaid in white, the polariton branches obtained from the Hamiltonian $H$ (eqs 1 and 2) in color. The avoided crossing around the heavy hole transition is resolved fully with a Rabi splitting of $66 \pm 1 \mathrm{meV}$, the broad line width of the higher energy light hole exciton prevents the resolution of the corresponding splitting, but mode position and intensity clearly indicate the coupling. (c, d) Transmission spectra for cavity lengths $L=1.528 \mu \mathrm{m}$ and $L=1.440 \mu \mathrm{m}$, respectively, and fits with two Lorentzians revealing the Rabi splitting for the heavy hole and (not fully resolved) light hole transition. (e) Square of coefficients $\alpha$, $\beta$, and $\gamma$ showing the hybridization of photonic mode and excitonic transitions as obtained from diagonalization of eq 2. The three different subplots correspond to the upper (UP), middle (MP), and lower (LP) polariton branches (from top to bottom).

$2 V_{\mathrm{hh}(\mathrm{lh})}$. In our system, the splitting is well resolved for the heavy hole exciton, where we obtain $\hbar \Omega_{\mathrm{hh}}=66 \pm 1 \mathrm{meV}$ with line widths of $\Gamma_{\mathrm{lp}}=35 \pm 3 \mathrm{meV}$ and $\Gamma_{\mathrm{mp}}=45 \pm 3 \mathrm{meV}$ for lower and middle polariton branch, respectively. The light hole transition shows a similar avoided crossing with $\hbar \Omega_{\mathrm{lh}}=58 \pm 4$ $\mathrm{meV}$, but the corresponding line widths of $\Gamma_{\mathrm{mp}}=68 \pm 13 \mathrm{meV}$ and $\Gamma_{\text {up }}=83 \pm 14 \mathrm{meV}$ for middle and upper polariton branch, respectively, result in a splitting that is not fully resolved. The line widths and splitting values are obtained by fitting two superposed Lorentzian lineshapes to the transmission spectra at the maximal photon-exciton mixing point (see Figure $2 \mathrm{c}-\mathrm{d}$ ), which corresponds to vertical cuts through the data presented in Figure 2 , panel a at cavity lengths $L \approx 1.53 \mu \mathrm{m}$ and $L \approx 1.44$ $\mu \mathrm{m}$. More cavity transmission spectra are shown in Figure $\mathrm{S} 1$ in the Supporting Information.

Eq 2 allows us to determine the polariton mixing coefficients as a function of cavity length, as shown in Figure 2, panel e. For a longitudinal cavity mode with mode number $q$ (in Figure $2 \mathrm{a}-$ e we have $q=6$ ), the LPB is largely photon-like for cavity lengths $L_{\mathrm{cav}}>1.53 \mu \mathrm{m}$ and becomes more hh exciton-like as the cavity mode energy crosses the exciton energy. The UPB similarly is lh exciton-like when the cavity length $L_{\text {cav }}>1.44 \mu \mathrm{m}$ and becomes more photon-like for smaller cavity lengths. Meanwhile, the MPB has hh and lh exciton-like character when close in energy to the hh and lh exciton energies, respectively. For cavity lengths $1.53 \mu \mathrm{m}>L_{\text {cav }}>1.44 \mu \mathrm{m}$, it has mostly photonic character; however, it also is composed of approximately $10 \%$ of both exciton states; hence, it is called a hybridized polariton state. We note that in a transmission experiment, light couples into and out of the cavity via the polariton's photon component, which results in greater visibility of photon-like polariton states and observation of a mode structure as shown in Figure 2, panel a.

If the number of randomly aligned platelets $N$ coupled to the mode is known, it is possible to deduce the transition dipole moment $\mu$ of a single platelet from the equation: ${ }^{24}$

$$
\hbar \Omega=\frac{1}{3} \mu \sqrt{N}\left(\frac{2 \hbar \omega}{\varepsilon_{0} n_{\mathrm{eff}}^{2} V_{\mathrm{eff}}}\right)^{1 / 2}
$$

Here, $\hbar \omega$ is the exciton energy, $n_{\text {eff }}$ is the effective refractive index within the cavity, and $V_{\text {eff }}$ is the electric field mode volume. We can then infer the dipole moment of the excitonic transition of a single platelet from the respective Rabi splitting. We find (see Supporting Information) 

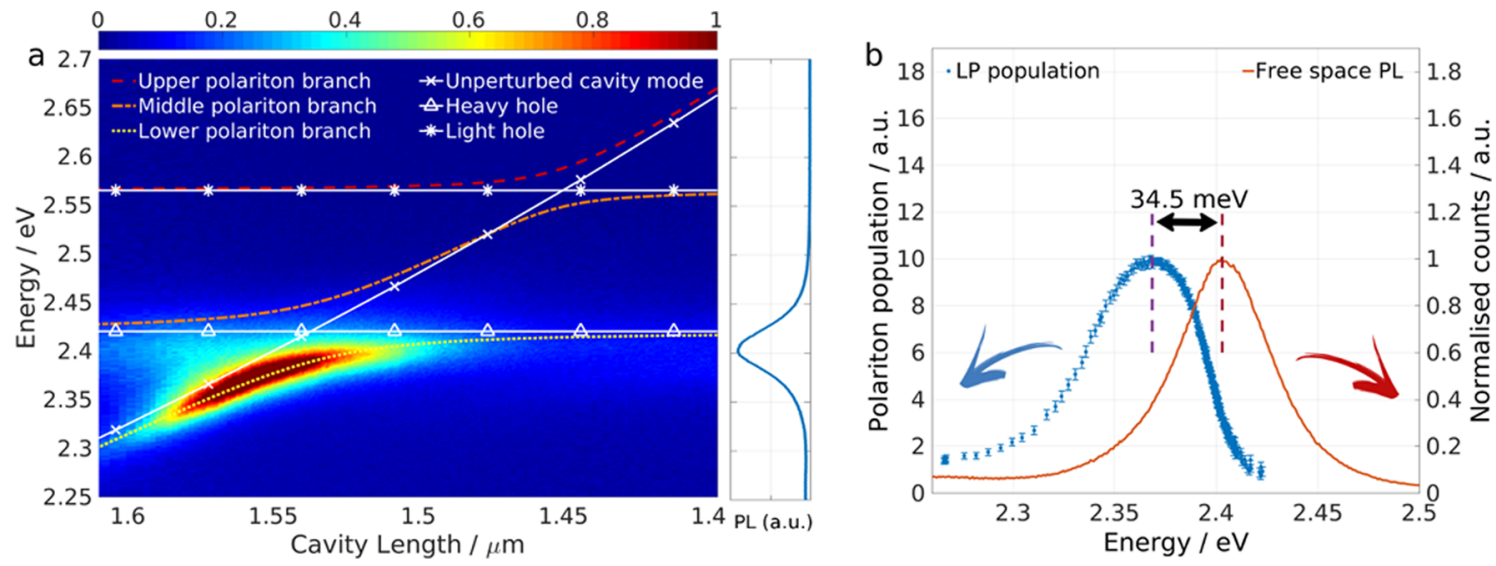

Figure 3. (a) Photoluminescence intensity of polariton branches as cavity length is varied. The sample is excited off-resonantly with a continuous wave laser with $\lambda=405 \mathrm{~nm}$. (b) Normalized polariton population (blue, circles with errorbar) and free space PL (red, continuous) obtained from experimental data by fitting each spectrum with a sum of two Lorentzian lineshapes and scaling the intensity of the lower polariton state with the corresponding inverse of the square of the photonic coefficient $\alpha$. A shift of $\Delta E=34.5 \mathrm{meV}$ between the free space PL peak position and the maximum polariton population is apparent, which results from energy lost during the inelastic scattering of excitons into the lower polariton state.

$$
\begin{gathered}
N=(3.9 \pm 1.5) \times 10^{5} \\
\mu_{\text {hh }}=(1.92 \pm 0.37) \times 10^{-27} \mathrm{Cm}=(575 \pm 110) \mathrm{D}
\end{gathered}
$$

This compares to transition dipole moments of $100 \mathrm{D}$ found in epitaxially grown GaAs quantum dots ${ }^{25}$ and 21 to $210 \mathrm{D}$ in InGaN quantum dots with a diameter of $5.2 \mathrm{~nm}^{26}$ The lateral dimensions of the platelets studied here are $L_{x}=32.5 \pm 2.5 \mathrm{~nm}$ and $L_{y}=8.2 \pm 0.9 \mathrm{~nm}$ as obtained by TEM microscopy. The transition dipole moment $\mu$ translates to a lifetime $\tau$ of the heavy hole exciton of $\tau=(1.3 \pm 0.5)$ ps. Similarly, the oscillator strength $f$ of the transition can be found as $f=(280 \pm 107)$ (see Supporting Information eqs 9 and 10). These results confirm the giant oscillator strength associated with the large exciton coherence area found in CdSe nanoplatelets. ${ }^{23,27}$

Figure 2, panel $b$ shows the system dispersion in a series of transmission spectra obtained by transfer-matrix-modeling (TMM) with the experimental absorption data for the nanoplatelet film as an input. The modeled thickness of the film is $700 \mathrm{~nm}$ (see Supporting Information for AFM data) with a peak absorbance of the heavy hole transition of $A=0.35$, as inferred directly from optical absorption measurement. The absorption curve was converted to complex refractive index values with a classical Lorentz oscillator model ${ }^{28}$ for both transitions, which neglected the continuum of states above $E=$ $2.8 \mathrm{eV}$. The results of the TMM calculations are in excellent agreement with the experimental data presented in Figure 2, panel a.

Figure 3, panel a shows the cavity length dependent photoluminescence (PL) collected at normal incidence, which follows nonresonant excitation with a continuous wave laser with $\lambda=405 \mathrm{~nm}$. We observe bright emission from the lower polariton branch at cavity lengths between 1.6 and $1.5 \mu \mathrm{m}$, corresponding to a LPB energy of 2.3 to $2.4 \mathrm{eV}$. Some weak residual PL is also visible at the energy of the nanoplatelet PL (shown to right of Figure 3a). This signal results from the recombination of excitons that are not coupled to the cavity mode, some of which leak directly through the mirrors. Emission from the MPB and UPB is not visible, as has previously been shown in other systems to be a result of fast relaxation pathways between polariton and lower lying exciton

states. ${ }^{29}$ Scaling the photoluminescence intensity of the lower polariton state (i.e., the area below the peak, as discussed in Figure S4 in the Supporting Information) with the square of the inverse of the photonic coefficient $\alpha$ of the polariton branch allows us to see changes in the polariton population along the dispersion, as shown in Figure 3, panel b, blue circles with error bars. A shift of $\Delta E=34.5 \mathrm{meV}$ between the peak position of free space PL and polariton population is visible. We distinguish two different population pathways for the polariton state: optical pumping and thermally assisted scattering. In optical pumping, the radiative decay of uncoupled excitons directly populates the photonic component of polariton states; however, there must be an energetic overlap between the exciton emission and polariton, and a fraction of the excitons within the cavity must remain uncoupled. The small Stokes shift of $12 \mathrm{meV}^{23}$ therefore limits this population mechanism to polariton states close to the exciton energy. ${ }^{30-32}$ Thermally assisted relaxation into the polariton state is akin to normal band-edge relaxation and relies on a phonon bath to absorb the additional energy. The coupling to the phonon background is proportional to the square of the excitonic component of the polariton state. ${ }^{33,34}$ The large shift $\Delta E$ observed in our system therefore suggests that polariton states are primarily populated by phonon-assisted scattering rather than through "optical pumping" from uncoupled exciton decay.

We have demonstrated the strong coupling between photonic cavity modes and excitons in quasi-two-dimensional colloidal nanoplatelets. The coherent exchange of energy between those two constituents results in the formation of hybridized exciton-polaritons at room temperature with vacuum Rabi splittings of $66 \pm 1 \mathrm{meV}$ and $58 \pm 1 \mathrm{meV}$ associated with the heavy hole and light hole exciton transitions, respectively. We find that the lower polariton state is emissive and primarily populated by phonon-assisted scattering. Nanoplatelets represent a promising candidate for polariton-based devices due to their large exciton binding energies that allow for room temperature operation. Compared to other colloidal nanoparticles, they are more efficient light absorbers and could become an integral part of future photonic devices. 


\section{ASSOCIATED CONTENT}

\section{S Supporting Information}

The Supporting Information is available free of charge on the ACS Publications website at DOI: 10.1021/acs.nanolett.6b03433.

Original data sets available at https://ora.ox.ac.uk/ (PDF)

\section{AUTHOR INFORMATION}

\section{Corresponding Authors}

*E-mail: lucas.flatten@materials.ox.ac.uk.

*E-mail: jason.smith@materials.ox.ac.uk.

\section{Notes}

The authors declare no competing financial interest.

\section{ACKNOWLEDGMENTS}

We thank Radka Chakalova at the Begbroke Science Park for helping with the thermal evaporation and dicing of the mirrors. L.F. acknowledges funding from the Leverhulme Trust. D.M.C. acknowledges funding from the Oxford Martin School and EPSRC Grant No. EP/K032518/1.

\section{REFERENCES}

(1) Weisbuch, C.; Nishioka, M.; Ishikawa, A.; Arakawa, Y. Phys. Rev. Lett. 1992, 69, 3314-3317.

(2) Lidzey, D. G.; Bradley, D. D. C.; Skolnick, M. S.; Virgili, T.; Walker, S.; Whittaker, D. M. Nature 1998, 395, 53-55.

(3) Agranovich, V. M.; Litinskaia, M.; Lidzey, D. G. Phys. Rev. B: Condens. Matter Mater. Phys. 2003, 67, 085311.

(4) Semond, F.; Sellers, I. R.; Natali, F.; Byrne, D.; Leroux, M.; Massies, J.; Ollier, N.; Leymarie, J.; Disseix, P.; Vasson, A. Appl. Phys. Lett. 2005, 87, 021102.

(5) Christmann, G.; Butté, R.; Feltin, E.; Mouti, A.; Stadelmann, P. A.; Castiglia, A.; Carlin, J.-F.; Grandjean, N. Phys. Rev. B: Condens. Matter Mater. Phys. 2008, 77, 085310.

(6) van Vugt, L. K.; Rühle, S.; Ravindran, P.; Gerritsen, H. C.; Kuipers, L.; Vanmaekelbergh, D. Phys. Rev. Lett. 2006, 97, 147401.

(7) Shimada, R.; Xie, J.; Avrutin, V.; Özgür, U.; Morkoč, H. Appl. Phys. Lett. 2008, 92, 011127.

(8) Liu, X.; Galfsky, T.; Sun, Z.; Xia, F.; Lin, E.-c.; Lee, Y.-H.; KénaCohen, S.; Menon, V. M. Nat. Photonics 2014, 9, 30-34.

(9) Flatten, L. C.; He, Z.; Coles, D. M.; Trichet, A. A. P.; Powell, A. W.; Taylor, R. A.; Warner, J. H.; Smith, J. M. Sci. Rep. 2016, 6, 33134. (10) Giebink, N. C.; Wiederrecht, G. P.; Wasielewski, M. R. Appl. Phys. Lett. 2011, 98, 081103.

(11) Christopoulos, S.; von Högersthal, G. B. H.; Grundy, A. J. D.; Lagoudakis, P. G.; Kavokin, A. V.; Baumberg, J. J.; Christmann, G.; Butté, R.; Feltin, E.; Carlin, J.-F.; Grandjean, N. Phys. Rev. Lett. 2007, 98, 126405.

(12) Kéna-Cohen, S.; Forrest, S. R. Nat. Photonics 2010, 4, 371-375.

(13) Rodriguez, S. R. K.; Amo, A.; Sagnes, I.; Le Gratiet, L.; Galopin, E.; Lemaitre, A.; Bloch, J. Nat. Commun. 2016, 7, 11887.

(14) Amo, A.; Lefrère, J.; Pigeon, S.; Adrados, C.; Ciuti, C.; Carusotto, I.; Houdré, R.; Giacobino, E.; Bramati, A. Nat. Phys. 2009, $5,805-810$.

(15) Byrnes, T.; Kim, N. Y.; Yamamoto, Y. Nat. Phys. 2014, 10, 803813.

(16) Ekimov, A. I.; Efros, A. L.; Onushchenko, A. A. Solid State Commun. 1985, 56, 921-924.

(17) Yin, Y.; Alivisatos, A. P. Nature 2005, 437, 664-670.

(18) Guyot-Sionnest, P. C. R. Phys. 2008, 9, 777-787.

(19) Carbone, L.; et al. Nano Lett. 2007, 7, 2942-2950.

(20) Ithurria, S.; Dubertret, B. J. Am. Chem. Soc. 2008, 130, 1650416505 .
(21) Ithurria, S.; Tessier, M. D.; Mahler, B.; Lobo, R. P. S. M.; Dubertret, B.; Efros, A. L. Nat. Mater. 2011, 10, 936-941.

(22) Tessier, M. D.; Javaux, C.; Maksimovic, I.; Loriette, V.; Dubertret, B. ACS Nano 2012, 6, 6751-6758.

(23) Naeem, A.; Masia, F.; Christodoulou, S.; Moreels, I.; Borri, P.; Langbein, W. Phys. Rev. B: Condens. Matter Mater. Phys. 2015, 91, 121302 .

(24) Fox, M. Quantum Optics: An Introduction; OUP Oxford, 2006.

(25) Guest, J. R.; Stievater, T. H.; Li, X.; Cheng, J.; Steel, D. G.; Gammon, D.; Katzer, D. S.; Park, D.; Ell, C.; Thränhardt, A.; Khitrova, G.; Gibbs, H. M. Phys. Rev. B: Condens. Matter Mater. Phys. 2002, 65, 241310.

(26) Ostapenko, I. A.; Hönig, G.; Kindel, C.; Rodt, S.; Strittmatter, A.; Hoffmann, A.; Bimberg, D. Appl. Phys. Lett. 2010, 97, 063103.

(27) Achtstein, A. W.; Scott, R.; Kickhöfel, S.; Jagsch, S. T.; Christodoulou, S.; Bertrand, G. H.; Prudnikau, A. V.; Antanovich, A.; Artemyev, M.; Moreels, I.; Schliwa, A.; Woggon, U. Phys. Rev. Lett. 2016, 116, 116802

(28) Fox, M. Optical Properties of Solids, Ch. 2; Oxford University Press, 2010

(29) Virgili, T.; Coles, D.; Adawi, A. M.; Clark, C.; Michetti, P.; Rajendran, S. K.; Brida, D.; Polli, D.; Cerullo, G.; Lidzey, D. G. Phys. Rev. B: Condens. Matter Mater. Phys. 2011, 83, 245309.

(30) Lidzey, D. G.; Fox, A. M.; Rahn, M. D.; Skolnick, M. S.; Agranovich, V. M.; Walker, S. Phys. Rev. B: Condens. Matter Mater. Phys. 2002, 65, 195312.

(31) Litinskaya, M.; Reineker, P.; Agranovich, V. M. J. Lumin. 2004, 110, 364-372.

(32) Litinskaya, M.; Reineker, P.; Agranovich, V. M. J. Lumin. 2006, 119-120, 277-282.

(33) Tassone, F.; Piermarocchi, C.; Savona, V.; Quattropani, A.; Schwendimann, P. Phys. Rev. B: Condens. Matter Mater. Phys. 1997, 56, 7554-7563.

(34) Michetti, P.; La Rocca, G. C. Phys. Rev. B: Condens. Matter Mater. Phys. 2009, 79, 035325. 